\title{
Impact of the GE1/1 station on the performance of the muon system in CMS
}

\author{
A. Magnani on behalf of the CMS GEM Collaboration ${ }^{\mathrm{a}, *}$
}

${ }^{a}$ INFN Pavia and University of Pavia, Pavia, Italy

\begin{abstract}
In 2018 the a new muon station based on GEM detectors will be installed in the CMS experiment. The present work sums up the main results obtained to evaluate the impact of this upgrade on the muon trigger rate and on muon reconstruction.
\end{abstract}

Keywords: Muon detectors, GEM, Micro-pattern Gas Detectors, CMS

PACS: 29.40.Cs, 29.40.Gx

\section{Introduction}

The muon spectrometer of the Compact Muon Solenoid experiment was designed as an almost hermetic and redundant system relying on different ionization detectors. Four stations of Drift Tubes (DT) in the barrel and Cathode Strip Chambers (CSC) in the endcaps provide position measurement and triggering. In addition, Resistive Plate Chambers (RPC), with their coarser granularity but fast timing properties, guarantee trigger redundancy in all the spectrometer, apart from the forward endcap region that covers the pseudorapidity range $1.6<|\eta|<2.4$ [1]. As a matter of fact, this region has background rates at the limit of the RPC rate capability (up to $1 \mathrm{kHz} / \mathrm{cm}^{2}$ ), therefore it is equipped solely with CSC chambers. The high $\eta$ region not only has the least redundancy, but it is also the most challenging for muon triggering and reconstruction [2] due to the exceptionally high rates and the reduced magnetic field.

After the upgrades planned for the Long Shutdowns LS2 ${ }^{33}$ and LS3 foreseen in 2018 and 2023, the Large Hadron Col- ${ }^{34}$ lider luminosity will exceed the design value by a factor two ${ }^{35}$ and five respectively. Maintaining efficient muon triggering in ${ }^{36}$ the forward region after the LHC upgrades represents a par- ${ }^{37}$ ticular challenge. Already at an instantaneous luminosity of ${ }^{38}$ $1.7 \times 10^{34} \mathrm{~cm}^{-2} \mathrm{~s}^{-1}$ the rate of the single muon trigger in this re- ${ }^{39}$ stricted part of the muon system is expected to approach values ${ }^{40}$ of $10 \mathrm{kHz}$, corresponding to one tenth of the entire Level- 1 trig- ${ }^{41}$ ger bandwidth [3] [4]. The trigger rate could be reduced simply by increasing the muon transverse momentum $p_{T}$ threshold, but this would deeply affect the sensitivity of all the physics 43 analyses that include soft muons in their signatures.

\section{The GE1/1 muon station}

In order to keep reasonable trigger rate with not too high $p_{T}{ }_{48}^{47}$ thresholds in the future LHC operation phases, the CMS GEM ${ }_{49}$

\footnotetext{
${ }^{*}$ Corresponding author

Email address: alice.magnani@cern.ch (A. Magnani on behalf of the 52
} CMS GEM Collaboration)

Preprint submitted to Elsevier

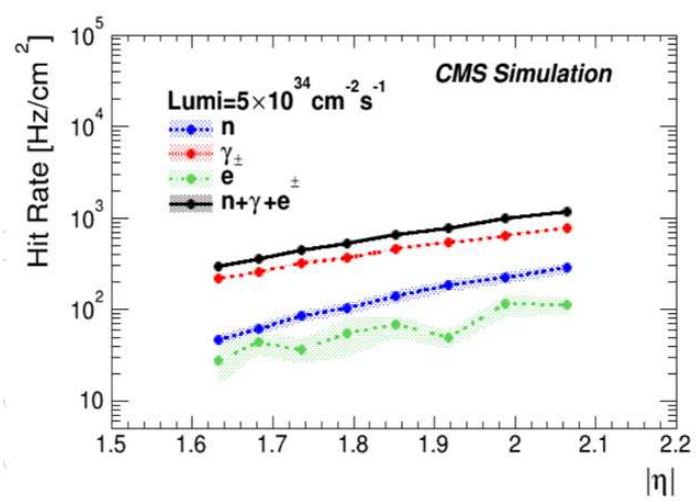

Figure 1: The expected background contribution to the GE1/1 detector hit rate for instantaneous luminosity of $5 \times 10^{34} \mathrm{~cm}^{-2} \mathrm{~s}^{-1}$ as a function of pseudorapidity.

Collaboration has planned to install during LS2 an additional measurement station, referred to as GE1/1. It will be based on the Gas Electron Multipliers (GEM) technology [5] and it will cover the pseudorapidity range $1.6<|\eta|<2.2$. Remarkable efforts have been spent to develop triple-GEM prototypes with high spatial and time resolution and high rate capability. The background rate expected in the GE1/1 region is shown in Figure 1 and it is orders of magnitude below the rate capability of the chambers, whose gain has been proved to be stable up to $100 \mathrm{MHz} / \mathrm{cm}^{2}$ [3].

\subsection{Impact on the muon trigger rate}

The GE1/1 chambers will complement the operation of the CSCs installed in the first muon station called ME1/1. The muon lever-arm between the GEMs and the adjacent CSCs will allow to determine the muon $p_{T}$ by measuring the bending angle due to the magnetic field in the first muon station alone. This $p_{T}$ measurement, independent from the one based on the muon bending through the whole detector, helps in reducing the rate of soft muons that pass the trigger threshold due to $p_{T}$ mismeasurements. The consequent reduction in the trigger rate is shown in Figure 2. 


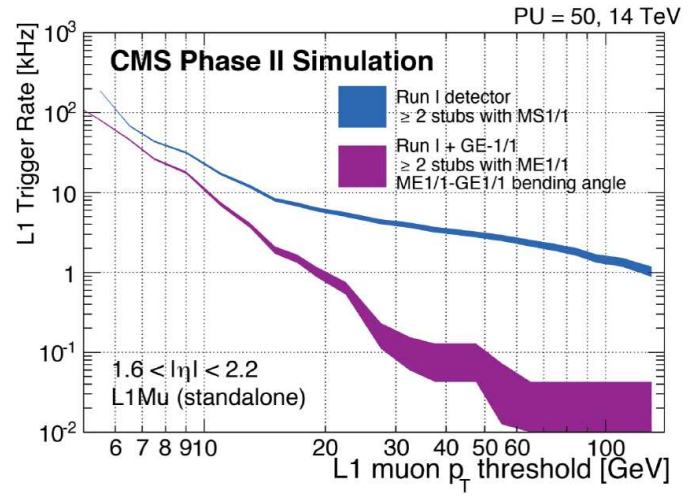

Figure 2: Level-1 muon trigger rates before and after the GE1/1 upgrade at a luminosity of $2 \times 10^{34} \mathrm{~cm}^{-2} \mathrm{~s}^{-1}$, for constant efficiency of $94 \%$

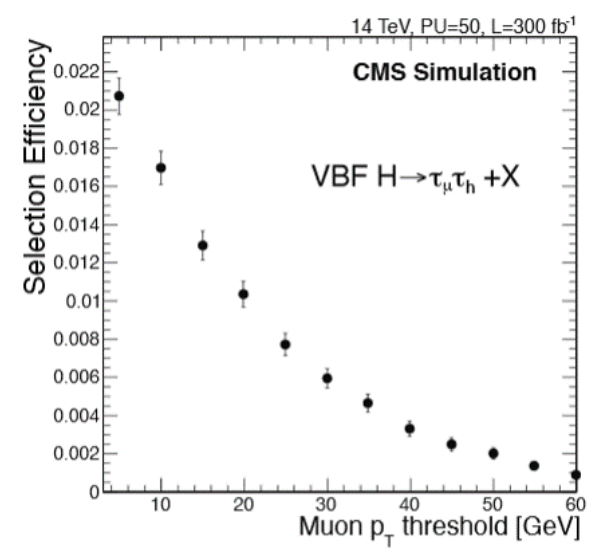

Figure 3: VBF $h \rightarrow \tau \tau$ analysis selection efficiency for the $\mu \tau_{h}$ final state as a 88 function of the chosen offline muon $p_{T}$ threshold.

The importance of keeping low $p_{T}$ thresholds has been investigated in various benchmark analyses, including the vector boson fusion higgs production with the subsequent decay to a pair 92 of $\tau$ leptons, one of them going to a muon and the other one decaying hadronically. A simulated sample of such events in post ${ }^{93}$ LS2 conditions was analyzed following kinematics and particle ${ }^{94}$ identification selections closely resembling requirements of the ${ }^{95}$ CMS Run $1 h \rightarrow 2 \tau$ observation paper [6]. One of the selection ${ }^{96}$ criteria is the presence of an high $p_{T}$ muon. Figure 3 shows how the selection efficiency behaves as a function of the muon ${ }_{97}$ $p_{T}$ threshold. A reduction from $25 \mathrm{GeV}$ to $15 \mathrm{GeV}$ yields a $68 \%$ increase in the number of signal events passing the selec- ${ }^{98}$ tions [3]

\subsection{Benefits to redundancy and reconstruction}

In the High Luminosity LHC phase starting in 2023, the tou CSCs installed in the forward region will have been operating ${ }^{105}$ for 14 years in an extreme radiation environment. Possible age- ${ }^{106}$ ing effects must be taken into account.

The solid line in Figure 4 illustrates how the trigger efficiency 109 is expected to decrease with the number of damaged CSCs in the first muon station. The same plot demonstrates how the integration of GE1/1 in the trigger system allows to devise algorithms that mitigate efficiency losses due to the presence of

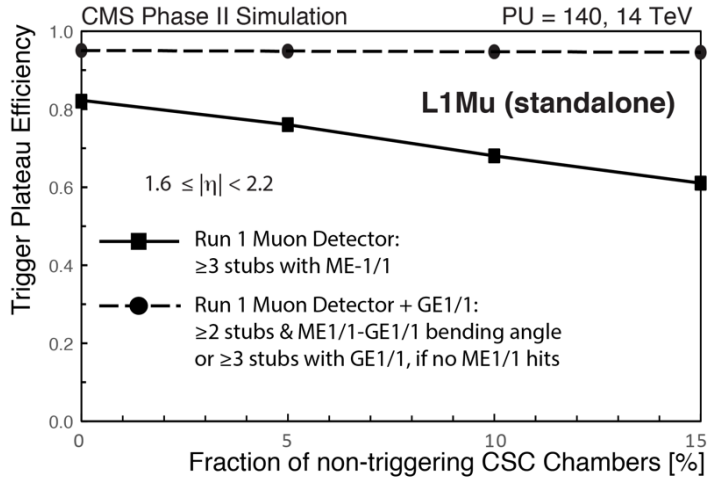

Figure 4: Single muon trigger efficiency at the plateau in $p_{T}$ as a function of the fraction of non triggering CSC chambers with and without the GE1/1 system.

\section{Acknowledgments}

We gratefully acknowledge support from FRS-FNRS (Belgium), FWO-Flanders (Belgium), BSF-MES (Bulgaria), BMBF (Germany), DAE (India), DST (India), INFN (Italy), NRF (Korea), QNRF (Qatar), and DOE (USA).

\section{References}

[1] CMS Collaboration: The CMS experiment at the CERN LHC, JINST 5 (2010) T03001.

[2] CMS Collaboration, Performance of CMS muon reconstruction in pp collision events at $\sqrt{s}=7 \mathrm{TeV}$, JINST 7 (2012) P10002

[3] CMS GEM Collaboration, CMS Technical Design Report for the muon endcap GEM upgrade, CMS-TDR-15-001-001.

[4] CMS Collaboration, Technical Proposal for the Phase-II Upgrade of the CMS Detector, CERN-LHCC-2015-010/LHCC-P-008.

[5] F. Sauli, GEM: A new concept for electron amplification in gas detectors, Nucl. Instrum. Meth. A386 (1997) 531.

[6] CMS Collaboration, Evidence for the $125 \mathrm{GeV}$ Higgs boson decaying to a pair of $\tau$ leptons, J. High Energy Phys. 104 (2014). 